\title{
Automated and accurate assessment for postural abnormalities in patients with Parkinson's disease based on Kinect and machine learning
}

Zhuoyu Zhang ${ }^{1 \dagger}$, Ronghua Hong ${ }^{1 \dagger}$, Ao Lin' ${ }^{1}$, Xiaoyun Su², Yue Jin ${ }^{2}$, Yichen Gao ${ }^{2}$, Kangwen Peng ${ }^{1}$, Yudi Li², Tianyu Zhang ${ }^{1}$, Hongping Zhi ${ }^{2}$ Qiang Guan ${ }^{1 *}$ and LingJing Jin ${ }^{1,3^{*}}$

\begin{abstract}
Background: Automated and accurate assessment for postural abnormalities is necessary to monitor the clinical progress of Parkinson's disease (PD). The combination of depth camera and machine learning makes this purpose possible.
\end{abstract}

Methods: Kinect was used to collect the postural images from 70 PD patients. The collected images were processed to extract three-dimensional body joints, which were then converted to two-dimensional body joints to obtain eight quantified coronal and sagittal features (F1-F8) of the trunk. The decision tree classifier was carried out over a data set established by the collected features and the corresponding doctors' MDS-UPDRS-III 3.13 (the 13th item of the third part of Movement Disorder Society-Sponsored Revision of the Unified Parkinson's Disease Rating Scale) scores. An objective function was implanted to further improve the human-machine consistency.

Results: The automated grading of postural abnormalities for PD patients was realized with only six selected features. The intraclass correlation coefficient (ICC) between the machine's and doctors' score was 0.940 (95\% Cl, 0.905-0.962), meaning the machine was highly consistent with the doctors' judgement. Besides, the decision tree classifier performed outstandingly, reaching $90.0 \%$ of accuracy, $95.7 \%$ of specificity and $89.1 \%$ of sensitivity in rating postural severity.

Conclusions: We developed an intelligent evaluation system to provide accurate and automated assessment of trunk postural abnormalities in PD patients. This study demonstrates the practicability of our proposed method in the clinical scenario to help making the medical decision about PD.

Keywords: Parkinson's disease, Postural abnormalities, Kinect, Machine learning

*Correspondence: guanqianglu@126.com; lingjingjin@tongji.edu.cn ${ }^{\dagger}$ Zhuoyu Zhang and Ronghua Hong contributed equally to this work ${ }^{1}$ Neurological Department of Tongji Hospital, Tongji University School of Medicine, Shanghai, China

${ }^{3}$ Department of Neurorehabilitation, Yangzhi Rehabilitation Hospital (Shanghai Sunshine Rehabilitation Center), Tongji University School of Medicine, Shanghai, China

Full list of author information is available at the end of the article

\section{Background}

Parkinson's disease (PD) is the second most common chronic neurodegenerative disease after Alzheimer's disease, characterized by motor impairments with tremor, rigidity and akinesia/bradykinesia as cardinal symptoms [1]. Postural abnormalities are frequent and disabling complications of PD. A cross-sectional study involving $811 \mathrm{PD}$ patients showed the prevalence of postural 
abnormalities reached 21.5\% [2]. Common postural abnormalities in PD include sagittal abnormalities: camptocormia and anterocollis; coronal abnormalities: Pisa syndrome and scoliosis [3]. Abnormal postures cause pain and balance dysfunction, aggravating walking difficulties with important impacts on life quality $[2,4]$. At present, its pathogenesis is unclear and there is no recognized objective and quantitative assessment method. Early recognition and standardized management will be helpful to delay the progression of postural abnormalities in PD to avoid worse outcomes.

There are various manual methods for evaluating abnormal posture of PD, including clinical scales, inclinometer, wall goniometer and photo-based measurement [5-8]. The most commonly used scale for evaluating abnormal posture of PD is the 13th item of the third part of Movement Disorder Society-Sponsored Revision of the Unified Parkinson's Disease Rating Scale (MDS-UPDRSIII 3.13), which is categorized into a discrete scale of five classes of increasing severity [9]. However, this method mainly relies on doctors' subjective judgement, which brings obvious intra and inter-rater variability. Though the inclinometer and wall goniometer methods are inexpensive and easy to operate, they only provide limited features and the results are not accurate $[5,8,10]$. The photo-based measurement method is considered as the gold standard to measure postural abnormalities in PD [7]. Nevertheless, it is time-consuming and the reference anatomical sites are not consistent among different tests.

Device-assisted posture evaluation which adopts accelerometers, gyroscopes, and optoelectronic system for analysis, provides sensitive, objective and real-time assessments [11-13]. However, they require sensors with satisfactory sensitivity and stability, which can be expensive [14]. Besides, wearable sensors are often not user-friendly. Patient engagement with wearable sensors is modest, possibly due to the absence of meaningful feedback and troublesome wearing [15]. Moreover, the analytical methods are complicated [16]. Clinical and technical expertise are needed to eliminate 'clinical noise' in the data analysis. Systems that are based on depth camera might represent a valid solution to overcome both the high cost and encumbrance of wearable devices.

Kinect is a low-cost and non-contact tracking device developed by Microsoft [17]. Okada et al. adopted the Kinect-based system for in-home posture evaluation and visual feedback training [18]. However, the system was unable to assess the postural abnormality automatically. In the last decade, machine learning (ML) has been widely used in the medical field to help doctors in medical judgment and decision [19-23]. ML techniques have been used and compared for PD classification with Kinect depth cameras [24-26]. Several trials using Kinect for automated assessment of UPDRS limb tasks and postural instability have been reported with an accuracy ranging from $74.0 \%$ to $95 \%$ [25, 27-29], including finger tapping, fists opening and closing, pronation and supination, stomping, posture and standing. Ferraris et al. [27] analyzed the posture of 28 PD patients with a Kinect camera. They performed automated machine grading based on supervised classifier training and compared with the scores of two doctors. The intraclass correlation coefficient (ICC) between the two doctors' scores was 0.77 while the ICC between the machine and the doctor's grading was 0.74 which suggested the possibility of automated grading by the machine, but the performance was not satisfactory enough.

We tried to develop an intelligent system based on Kinect and machine learning for evaluating postural characteristics in PD patients and achieved accurate description and automated grading of postural abnormalities. Taking advantage of a larger data set, we introduced multi-dimensional features of trunk posture and used decision tree of supervised classifier with an objective function implanted to improve the human-machine consistency.

\section{Methods \\ Experimental subjects}

Consecutive patients diagnosed with PD in Tongji Hospital Affiliated to Tongji University from October 2018 to January 2020 were enrolled. The inclusion criteria were: (1) Meet the diagnostic criteria of clinically confirmed PD in the 2015 MDS Parkinson's disease clinical diagnostic criteria [9]; (2) Able to stand by oneself. The exclusion criteria were: (1) Suspected or diagnosed with Parkinson's superimposed syndrome or secondary Parkinson's syndrome; (2) Patients with deformities or injuries of lower limbs; (3) Patients with mental disorders or cognitive impairments.

Written informed consent was obtained from all participants, and the present study was approved by the Ethics Committee of Shanghai Tongji Hospital.

\section{Devices and experimental procedure}

In cooperation with iFLYTEK Suzhou Research Institute, a motion analysis device integrating a Kinect v2.0 depth camera (RGB $1920 \times 1080$ pixels @30fps, depth camera $512 \times 424$ pixels @30fps, 4-microphone linear phased array, Microsoft) was built. The Kinect camera was connected to an independent computer that ran a data capture program that saved the data generated during the recording process.

The recording process was as follows (Fig. 1): Patients were asked to stand directly in front of the Kinect camera at ease for $5 \mathrm{~s}$ and then actively correct their abnormal 

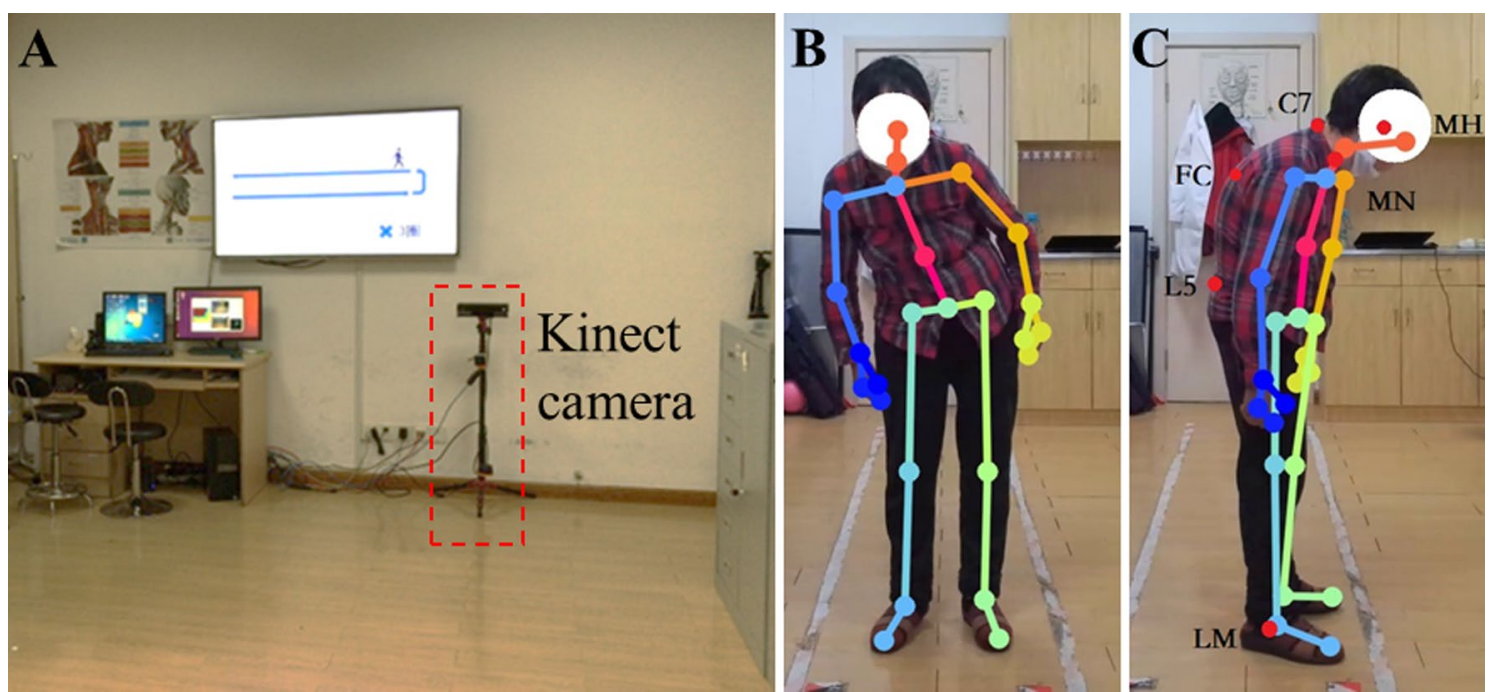

Fig. 1 Real image showing the test scene, in which you can see the Kinect camera $(\mathbf{A})$ and a participant undergoing the test (B and $\mathbf{C})$

posture for $5 \mathrm{~s}$. After that, they were asked to turn left for $90^{\circ}$, relax and stand for $5 \mathrm{~s}$, and then actively correct their abnormal posture for another $5 \mathrm{~s}$. Patients were advised to wear close-fitting clothing and long hair should be tied up. Their gender, age, onset age, length of disease and Hoehn-Yahr scale were collected and recorded. Meanwhile, 2 professionally trained doctors (namely doctor 1 and doctor2) individually rated the postural abnormalities of PD patients according to MDS-UPDRS-III 3.13.

\section{Data collection and processing}

According to previous reports combining clinical experience on the assessment methods of postural abnormalities, the collected abnormal posture features were defined as follows [7, 30, 31]: (1) F1:lateral flexion angle of head, the angle between the connecting line of midpoint of head $(\mathrm{MH})$ and midpoint of neck $(\mathrm{MN})$ on the coronal plane and the vertical line of the ground (VL) (Fig. 2A); (2) F2: lateral flexion angle of trunk, the angle between the connecting line of the 7 th cervical spinous process (C7) and the 5th lumbar spinous process (L5) on the coronal plane and VL (Fig. 2B); (3) F3: forward flexion angle of head, the angle between the connecting line of $\mathrm{MH}$ and $\mathrm{MN}$ on the sagittal plane and VL (Fig. 2C); (4) F4: total forward flexion angle of trunk, the angle between the connecting line of L5 and lateral malleolus (LM) and the connecting line of C7 and L5 on the sagittal plane (Fig. 2D); (5) F5: forward flexion angle of trunk at the waist, the angle between the connecting line of L5 and LM and the connecting line of vertebral fulcrum (FC, which indicates the most convex point of the vertebra) and L5 on the sagittal plane (Fig. 2E); (6) F6: forward flexion angle of trunk at the thorax, the angle between the connecting line of FC and L5 and the connecting line of C7 and FC on the sagittal plane (Fig. 2F); (7) F7: distance between the most convex point of the back and the trunk axis, the pixel distance between FC and the connecting line of C7 and L5 on the sagittal plane(Fig. 2G); (8) F8: forward flexion angle of head relative to trunk, the angle between the connecting line of $\mathrm{MH}$ and $\mathrm{MN}$ and the connecting line of C7 and L5 on the sagittal plane (Fig. $2 \mathrm{H}$ ).

Firstly, the Kinect depth map was used to segment the body part with the segmentation algorithm, which was conducive to the extraction of key points involved in features F1 to F8. The segmentation algorithm was set as follows:

$$
d_{\text {body }}(x, y)= \begin{cases}0 & \text { if abs }\left(d(x, y)-d_{c}\right)<t h r \\ 1 & \text { else }\end{cases}
$$

$d_{\text {body }}$ denoted the segmented body depth map, in which 0 was background, 1 was body part. $d$ represented the depth of a map. $\operatorname{abs}\left(d(x, y)-d_{c}\right)$ denoted the depth distance between the point $(x, y)$ and body center. thr was the threshold.

Then, the depth images collected by the Kinect camera were processed frame by frame, and a skeleton composed of 25 three-dimensional(3D) body joints was extracted using human skeleton and muscle model [24, 27]. Next, the $3 \mathrm{D}$ body joints were further projected to the two-dimensional(2D) body joints on a plane figure. Since these extracted joints were inconsistent with the key points actually used for feature calculation, the initial joints of Kinect skeleton were used as the basis to further calculate the exact bone points for posture evaluation 

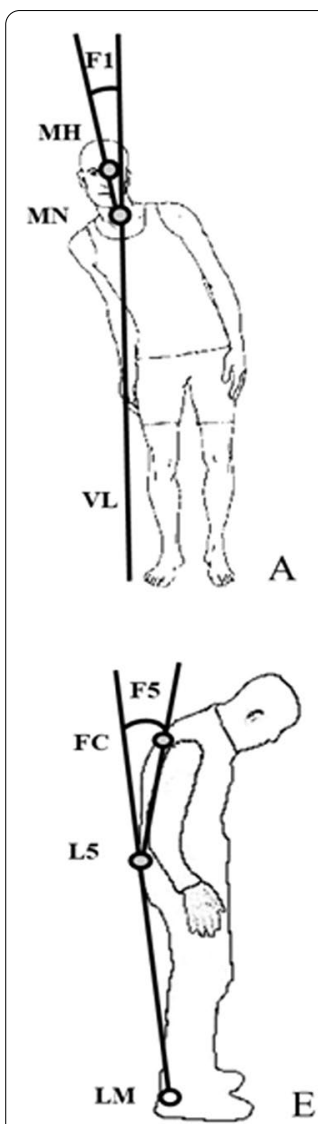
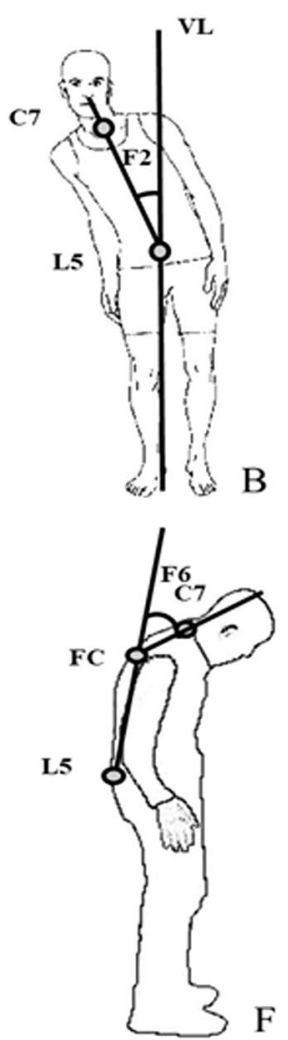
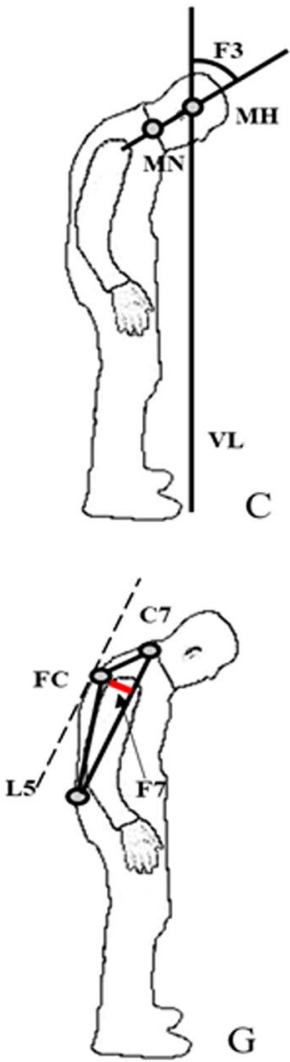
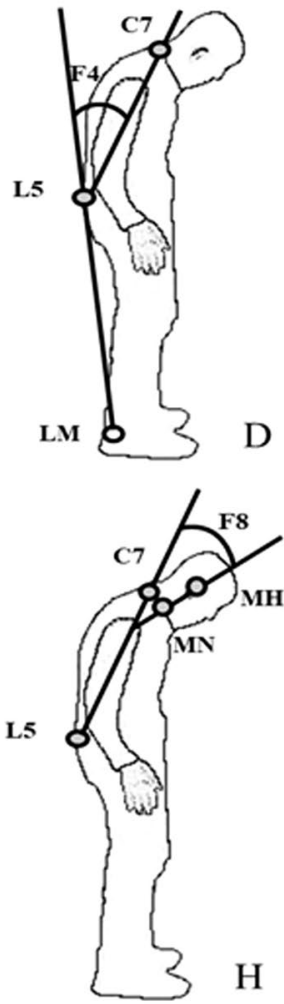

Fig. 2 Illustration of the measured features for abnormal trunk posture (A and B were modified from [4])

from a side view of the participant. Specifically, the head, neck and hip 2D joints of the Kinect skeleton were taken as benchmarks: we further segmented the segmented human body based on the morphological method by combining the coordinates of head and neck on the depth map to obtain the point $\mathrm{MH}$; taking the $2 \mathrm{D}$ coordinates of neck and hip on the depth map as the initial points, we got the contour points C7 and L5 along the back based on the depth point cloud. After that, we got FC by taking $\mathrm{C} 7$ and L5 as the benchmarks and combining the points on the entire back resulted from human body segmentation. In this way, a total of $302 \mathrm{D}$ points were acquired. Then, the pixel distance between any two of the points or the angle between any two lines connecting the points was automatically calculated to obtain the quantified kinematic features of the posture F1 to F8. Particularly, the feature F7 was normalized according to the height of the human body, eliminating the effects of height differences. After that, a supervised learning method named decision tree was carried out over a data set established by the collected features and the corresponding doctors' MDS-UPDRS-III 3.13 scores. An objective function was implanted to further improve the human-machine consistency. The objective function for minimizing calculation errors was set as follows:

$$
\begin{aligned}
& f=\text { mean }(\text { abs }(\text { doc }- \text { machine }))+ \\
& \lambda * \operatorname{len}(\text { find }(\text { abs }(\text { doc }- \text { machine })>1))
\end{aligned}
$$

The objective function $f$ consists of two parts. The first part is the deviation between the machine's and the doctor's score. The second part is the combination of the coefficient $\lambda$ and cases number, which error between the machine's and the doctors' score exceeds 1. Firstly, using Gini index as the segmentation criterion, a set of sub-optimal trees were trained. Then, the grid search was conducted to search for the optimal decision tree depth and split strategy ('best' or 'random') in the sub-optimal trees. In other words, the optimal decision tree is the smallest difference (not more than 1 as much as possible) between the machine's and the doctor's scores. Finally, 5 -fold cross-validation was performed on the sample set with expert annotations to avoid overfitting.

Metrics for evaluating machine learning performance such as accuracy, sensitivity, and specificity were obtained by applying the 5 -fold cross-validation 
method for the decision tree. We firstly transferred the 5 -class classification problem into five 2-class classification problems. The metrics for each 2 -class classifier were calculated respectively. Finally, the acquired metrics were averaged to estimate the metrics for the 5-class classifier.

\section{Statistical analysis}

Quantitative data were shown as mean \pm Standard Deviation (SD). The normality of distribution of demographic, clinical data and F1 to F8 were initially tested using the Kolmogorov-Smirnov test. The corresponding difference of the related data among the five different groups based on the results of MDS-UPDRSIII 3.13 score were analyzed using one-way analysis of variance (ANOVA) initially when the data followed normal distribution. Otherwise, Kruskal-Wallis H-test was adopted. Spearman correlation analysis was conducted to establish correlation between F1 to F8 and MDS-UPDRS-III 3.13 score. A $p$ value $<0.05$ indicated significant difference for all analysis. As we used multiple tests, $p$ value of intergroup comparison among the five different groups were corrected using the Bonferroni method. The alpha value was set at $p^{\prime}=0.05 /$ times of comparison that is $p^{\prime}=0.005$. We used ICC to calculate the consistency between the humanmachine score. According to previous literature, ICC values less than 0.5 are indicative of poor reliability, values between 0.5 and 0.75 indicate moderate reliability, values between 0.75 and 0.9 indicate good reliability, and values greater than 0.90 indicate excellent reliability [32].

\section{Results}

79 PD patients who completed the data and video collection from October 2018 to January 2020 were analyzed, and 9 of them were excluded due to poor image quality, large clothing and other factors. The postural features of the remaining 70 patients were automatically identified and accurately portrayed. The 70 patients were divided into group of ' 0 ', ' 1 ', ' 2 ', ' 3 ' and ' 4 ' respectively based on the results of MDS-UPDRS-III 3.13 score. The demographic, clinical data and F1 to F8 were shown in Table 1. A significant difference was found in age $(p=0.018)$, length of disease $(p=0.003)$, Hoehn-Yahr scale $(p=0.001), \quad$ F1 $\quad(p=0.010), \quad$ F2 $(p=0.008), \mathrm{F} 3(p<0.001), \mathrm{F} 4(p<0.001), \mathrm{F} 5(p<0.001)$, F6 $(p=0.013), \mathrm{F} 7(p<0.001)$ and F8 $(p=0.008)$ among groups of different MDS-UPDRS-III 3.13 score.

Compared with group '0', post hoc analysis found an increase of $\mathrm{F} 1 \quad(p=0.01)$ (Fig. 3A), F2 $(p=0.027)$ (Fig. 3B), F3 $(p<0.001)$ (Fig. 3C) and F8 $(p=0.01)$ (Fig. 3H) in group '2', an increase of F3 $(p<0.001)$ (Fig. 3C) and F4 $(p<0.001)$ (Fig. 3C) in group '4'. Compared with group ' 1 ', post hoc analysis found an increase of F3 $(p<0.001)$ (Fig. $3 \mathrm{C}), \mathrm{F} 4(p<0.001)$ (Fig. 3D), F5 $(p<0.001)$ (Fig. 3E) and F7 $(p<0.001)$ (Fig. 3G) in group '4.' Compared with group '2', post hoc analysis found an increase of F4 ( $p<0.001)$ (Fig. 3D), F5 $(p<0.001)$ (Fig. 3E) and F7 $(p<0.001)$ (Fig. 3G) in group '4. Compared with group ' 3 ', post hoc analysis found an increase of F4 $(p<0.001)$ (Fig. 3D) and F7 $(p<0.001)$ (Fig. 3G) in group ' 4 '.

We further conducted Spearman correlation analysis to explore correlations between F1 to F8 and MDS-UPDRS-III 3.13 score. The results were shown

Table 1 Demographic and clinical characteristics

\begin{tabular}{|c|c|c|c|c|c|c|}
\hline MDS-UPDRS-III 3.13 score & 0 & 1 & 2 & 3 & 4 & $p$-value \\
\hline Number & 13 & 12 & 33 & 6 & 6 & \\
\hline Male (\%) & $6(46.2)$ & $7(58.3)$ & $27(81.8)$ & $5(83.3)$ & $3(50.0)$ & 0.084 \\
\hline Age & $64.0 \pm 6.0$ & $70.3 \pm 7.4$ & $69.3 \pm 6.3$ & $62.0 \pm 11.2$ & $71.3 \pm 5.5$ & $0.018^{*}$ \\
\hline Onset age & $61.2 \pm 7.0$ & $67.5 \pm 8.3$ & $63.1 \pm 7.5$ & $57.3 \pm 10.8$ & $64.0 \pm 7.1$ & 0.170 \\
\hline Duration (years) & $2.8 \pm 2.8$ & $2.8 \pm 2.0$ & $6.2 \pm 3.6$ & $4.7 \pm 3.3$ & $7.3 \pm 5.5$ & $0.003^{* *}$ \\
\hline Hoehn-Yahr & $1.5 \pm 0.5$ & $1.8 \pm 0.8$ & $2.4 \pm 0.9$ & $2.7 \pm 0.5$ & $2.3 \pm 0.5$ & $0.001^{* *}$ \\
\hline F1 (degree) & $3.2 \pm 4.5$ & $5.8 \pm 4.2$ & $9.4 \pm 6.3$ & $10.3 \pm 12.0$ & $10.3 \pm 5.5$ & $0.010^{*}$ \\
\hline F2 (degree) & $0.7 \pm 0.9$ & $1.1 \pm 1.0$ & $2.3 \pm 1.8$ & $4.3 \pm 4.8$ & $2.3 \pm 1.6$ & $0.008^{* *}$ \\
\hline F3 (degree) & $26.5 \pm 8.0$ & $34.0 \pm 9.9$ & $42.6 \pm 9.3$ & $47.8 \pm 14.7$ & $54.5 \pm 19.0$ & $<0.001^{* * *}$ \\
\hline F4 (degree) & $18.7 \pm 3.4$ & $20.7 \pm 3.4$ & $23.9 \pm 4.9$ & $25.3 \pm 5.9$ & $41.8 \pm 15.1$ & $<0.001^{* * *}$ \\
\hline F5 (degree) & $8.4 \pm 4.0$ & $9.8 \pm 3.1$ & $11.9 \pm 5.7$ & $13.2 \pm 5.6$ & $25.8 \pm 18.9$ & $<0.001^{* * *}$ \\
\hline F6 (degree) & $31.1 \pm 5.5$ & $31.9 \pm 5.0$ & $36.0 \pm 6.5$ & $37.7 \pm 2.2$ & $39.7 \pm 6.5$ & $0.013^{*}$ \\
\hline F7 (\%) & $20.2 \pm 4.2$ & $22.1 \pm 3.3$ & $25.2 \pm 4.1$ & $26.8 \pm 4.0$ & $46.7 \pm 12.9$ & $<0.001^{* * *}$ \\
\hline F8 (degree) & $14.2 \pm 7.9$ & $19.8 \pm 9.0$ & $25.5 \pm 9.1$ & $29.2 \pm 12.1$ & $20.8 \pm 17.0$ & $0.008^{* *}$ \\
\hline
\end{tabular}

${ }^{*} p<0.05,{ }^{* *} p<0.01,{ }^{* * *} p<0.001$ 

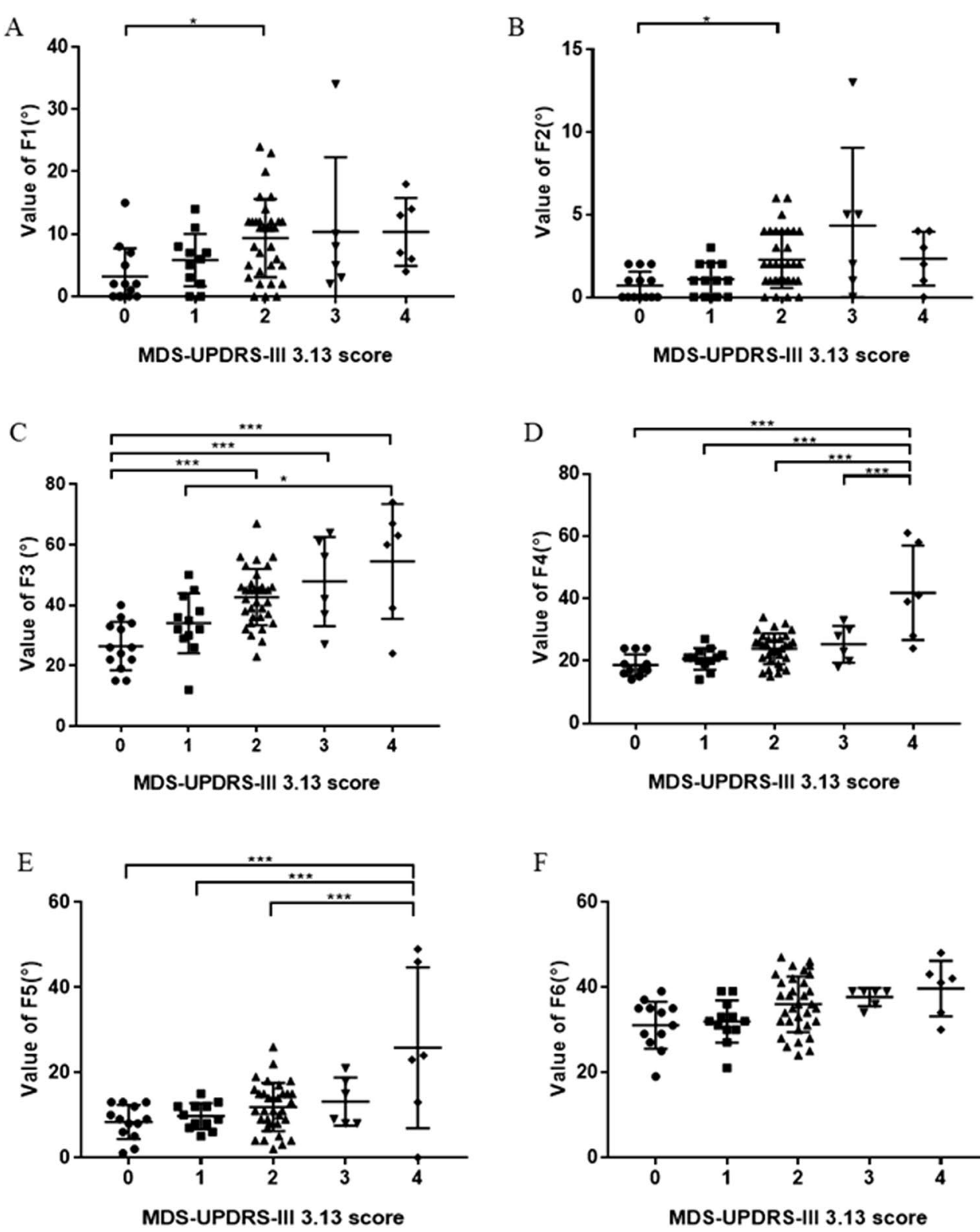

F
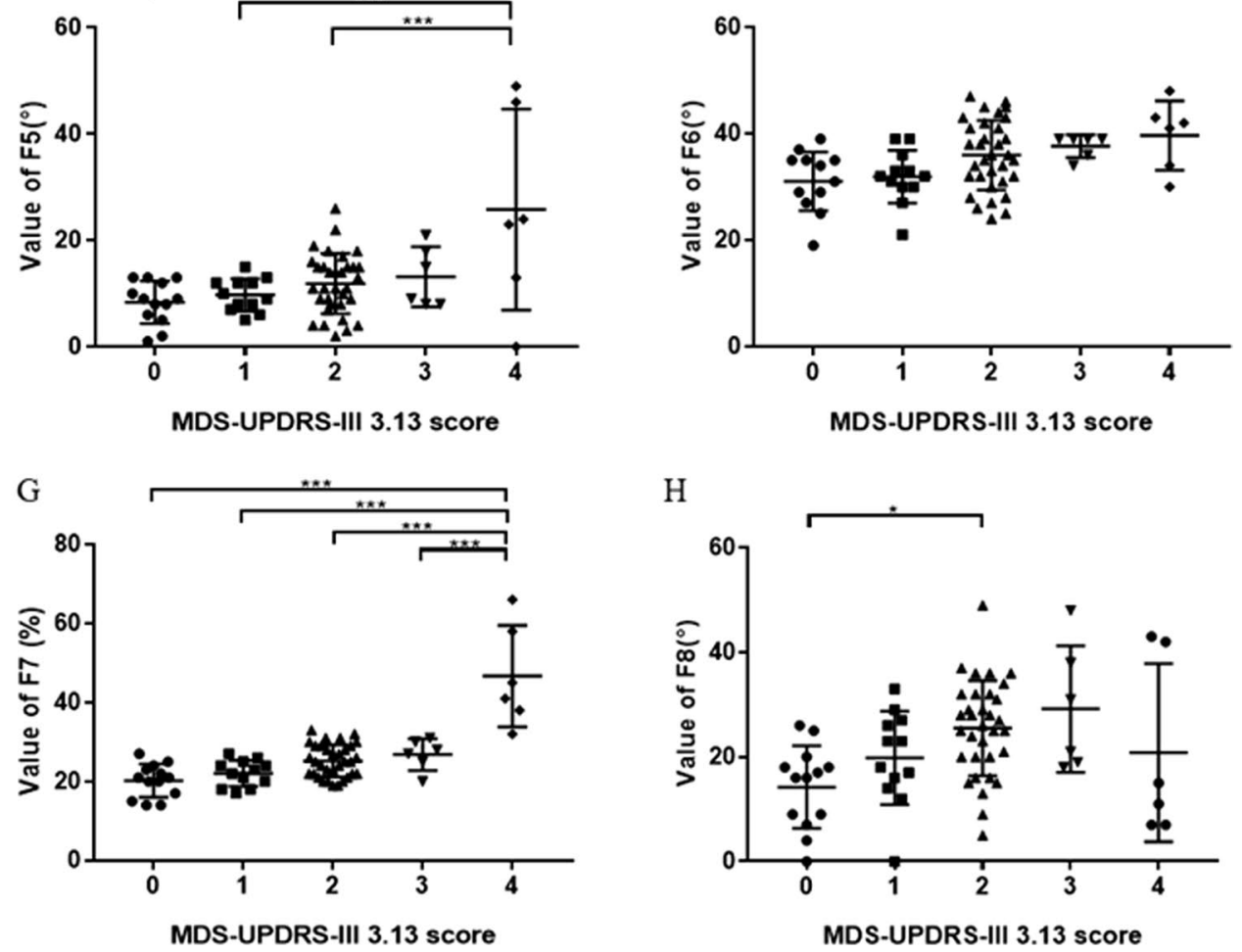

Fig. 3 Intergroup comparison of postural features among groups of different MDS-UPDRS-III 3.13 score 
Table 2 Correlation analysis between F1 to F8 and MDS-UPDRS-III 3.13 score

\begin{tabular}{llrrrrrrr}
\hline & F1 & F2 & F3 & F4 & F5 & F6 & F7 & F8 \\
\hline$r_{s}$ & 0.388 & 0.421 & 0.591 & 0.561 & 0.362 & 0.424 & 0.603 & 0.312 \\
$p$-value & 0.001 & $<0.001$ & $<0.001$ & $<0.001$ & 0.002 & $<0.001$ & $<0.001$ & 0.009 \\
\hline
\end{tabular}

$\mathrm{r}_{s}$ : Spearman correlation coefficient

Table 3 Selection results of the relevant features

\begin{tabular}{|c|c|c|}
\hline & $\mathrm{ICC}_{\mathrm{DD}}$ & $\mathrm{ICC}_{\mathrm{MD}}$ \\
\hline $\begin{array}{l}F 1+F 2+F 3+F 4+F 5+F 6 \\
\left(\max \text { depth }=5, \text { splitter }=\text { 'best' }^{\prime}\right)\end{array}$ & 0.814 & 0.707 \\
\hline $\begin{array}{l}F 1+F 2+F 3+F 4+F 5+F 7 \\
\left(\text { max depth }=5, \text { splitter }=\text { 'best' }^{\prime}\right)\end{array}$ & 0.814 & 0.786 \\
\hline $\begin{array}{l}\mathrm{F} 1+\mathrm{F} 2+\mathrm{F} 3+\mathrm{F} 4+\mathrm{F} 5+\mathrm{F} 6+\mathrm{F} 7+\mathrm{F} 8 \\
(\text { max depth }=5, \text { splitter }=\text { 'best') }\end{array}$ & 0.814 & 0.781 \\
\hline $\begin{array}{l}\mathrm{F} 1+\mathrm{F} 2+\mathrm{F} 3+\mathrm{F} 4+\mathrm{F} 5+\mathrm{F} 7 \\
(\text { max depth }=7, \text { splitter }=\text { 'random') }\end{array}$ & 0.814 & 0.940 \\
\hline
\end{tabular}

$\mathrm{ICC}_{\mathrm{DD}}$ : Intraclass correlation coefficient between two doctors

$\mathrm{ICC}_{\mathrm{MD}}$ : Intraclass correlation coefficient between the machine and doctor

in Table 2, which indicated that there was a significant positive correlation between F1 to F8 and MDSUPDRS-III 3.13 score.

The grid search and comparative experiments were conducted to search for the optimal combination of input features, decision tree depth and split strategy, which guaranteed the highest human-machine consistency. The selection results were shown in Table 3. The optimal model integrated the input features of F1, F2, F3, F4, F5 and F7, with a max depth of 7 and a split strategy of 'random.' Under this condition, the ICC between the machine's and the doctors' scores was 0.940 , which was much higher than the two doctors' ICC of 0.814 .

According to the optimal decision tree, the feature importance of the selected features to the machine's automated grading were $13.2 \%, 12.6 \%, 16.5 \%, 11.3 \%, 6.7 \%$ and $40 \%$ severally (Table 4 ).

Metrics for evaluating machine learning performance such as accuracy, sensitivity, and specificity were shown in Table 5. Besides, we trained the posture features based on Support Vector Machine (SVM) and k-Nearest Neighbours $(\mathrm{kNN})$ classifiers simultaneously. Their performance did not improve further.

\section{Discussion}

In the present study, we developed an intelligent evaluation system for postural abnormalities in PD based on Kinect and machine learning, enabling the multi-feature, accurate assessment and automated classification. The ICC between the machine's and doctors' score was 0.940 (95\%CI, 0.905-0.962). All the standard metrics of machine learning performed outstandingly with $90 \%$ of accuracy, $89 \%$ of sensitivity and $96 \%$ of specificity. According to a multicenter clinical trial, the test-retest reliability for the UPDRS-III score which was estimated by ICC was 0.90 . ICCs for derived symptom-based scales ranged from 0.69 to 0.88 [33]. Therefore, our system is sufficient for clinical application. To our knowledge, this study has the highest human-machine consistency related the automated grading of postural abnormalities in PD.

Ferraris analyzed the posture of $28 \mathrm{PD}$ patients with four features using Kinect [27]. They performed automated machine grading based on three supervised classifier training including kNN, SVM and Multinomial Logistic Regression (MLR). The ICC between the machine's and the doctor's grading was 0.74 and the classification accuracies range from 58.8 to $70.8 \%$. Our system developed a new generation with higher accuracy. Taking advantage of a larger data set, we derived more postural features for training the decision tree model. The coronal features (F1 and F2) and the sagittal features (F3, F4, F5 and F7) proved to be very relevant when classifying the PD stage related to posture in the obtained

Table 5 Classification metrics for the supervised classifiers

\begin{tabular}{llll}
\hline & Accuracy (\%) & Sensitivity (\%) & Specificity (\%) \\
\hline Optimal decision tree & $90.0 \%$ & 89.1 & 95.7 \\
SVM & 88.6 & 77.4 & 93.9 \\
kNN $(K=5)$ & 82.9 & 60.2 & 90.8 \\
\hline
\end{tabular}

Table 4 Importance of the selected features

\begin{tabular}{lllllll}
\hline & F1 & F2 & F3 & F4 & F5 & F7 \\
\hline Feature importance & $13.2 \%$ & $12.6 \%$ & $16.5 \%$ & $11.3 \%$ & $6.7 \%$ & $40.0 \%$ \\
\hline
\end{tabular}


decision tree model. Besides, we applied an objective function for minimizing calculation errors and searching for the optimal results [34, 35]. Both the deviation of the scores and the number of cases with obvious deviation (more than one score of MDS-UPDRS-III 3.13) were considered to ensure that there was less difference between the two scores even in the case where the machine's and the doctors' scores were inconsistent. In this way, the accuracy of the automated grading process was significantly improved.

Buongiorno et al. also used machine learning to analyze gait alterations of 14 healthy subjects and 16 PD patients collected by Kinect [28]. The results showed that the Artificial Neural Network (ANN) classifier performed well by reaching $89.4 \%$ of accuracy in discriminating PD from healthy control and $95.0 \%$ of accuracy in distinguishing mild or moderate patients (two-classes classifier, binary problem). However, it is not yet known how it performed in the finer classification (posture assessment in UPDRS is a five-classification problem). Our system focuses on postural abnormalities and aims to bring an automated and accurate assessment of UPDRS tasks, which can be used for posture assessment before and after medication, enabling dynamic monitoring of postural changes. All these provide effective assistance to doctors and patients. Furthermore, in the follow-up work, we are expanding the application areas of the system. For example, it will be applied to the automated analysis for fine movements of hands and feet, as well as the automated evaluation of sub-item and total score of UPDRS-III. This will bring great benefits to the accurate assessment of PD.

In addition to the above two studies, there are many researchers assessing posture based on Kinect [36-41]. However, machine learning and automated grading are not included, which make it fail to assess the postural abnormality automatically. Moreover, there are also a multitude of technology-based objective measures of postural abnormality that have been developed [14, 42, 43]. Asakawa et al. reviewed how the latest computerized technologies, including the wearable sensors, virtual reality, augmented reality, and robot assistant systems, improved the diagnosis and treatment of PD [14]. However, it is either complicated or expensive of the related equipment [16], which makes them hard to extend the user population and usage scenario. Furthermore, the wearable sensors need to be worn by the patient. Some patients will stand more erect after wearing sensors, which affect the evaluation of the postural abnormalities [44]. Our system is low-cost, non-contact and userfriendly. Patients can get results quickly without wearing any equipment. Besides, the system has simple hardware and software, which is suitable for multiple scenarios such as hospitals, homes and research institutes.
In this work, we found a significant strong correlation between F7 and MDS-UPDRS-III $3.13\left(\mathrm{r}_{\mathrm{s}}=0.603\right.$, $p<0.001)$. F7, which represents the pixel distance between FC and the connecting line of C7 and L5 on the sagittal plane, reflects well the severity of camptocormia in PD patients. Conventionally, three common methods, including total camptocormia method (deviates of C7/L5 line from vertical), upper camptocormia method (flexion between the lower thoracic spine and upper lumbar spine) and lower camptocormia method (flexion of hip) were used to assess camptocormia while they produced camptocormia angles with dramatical difference by up to $50 \%$ on the same patient [7]. It requires three parameters to describe the severity of camptocormia accurately. However, to our study, F7 per se accounted for the heaviest feature importance (40\%) in the established model, suggesting its key role for machine learning in distinguishing different postural degrees of camptocormia. It is the first time that F7 is proposed and gives a general assessment in patients with both lower and upper camptocormia. Further research shall be conducted to demonstrate whether this single index can serve as a useful and reliable feature to evaluate the severity of camptocormia, especially its relationship between clinical manifestations and life quality of PD patients.

Apart from F7, we also found a significant medium to strong correlation between F3 and MDS-UPDRS-III $3.13\left(\mathrm{r}_{\mathrm{s}}=0.591, p<0.001\right)$ and between F4 and MDSUPDRS-III $3.13\left(\mathrm{r}_{\mathrm{s}}=0.561, p<0.001\right)$. F3 represents forward flexion angle of head, reflecting the severity of cervical lordosis [5]. F4, which represents total camptocormia angle, was a reliable method proposed by Margraf [7]. Both F3 and F4 are valuable indexes for the severity of camptocormia. Though F6 and F8 were not included in the established decision tree model, it didn't necessarily mean these two features were not relevant to the severity of postural abnormalities since Spearman correlation analysis revealed both a significant positive correlation between F6 and MDS-UPDRS-III $3.13\left(\mathrm{r}_{\mathrm{s}}=0.424\right.$, $p<0.001)$ and between F8 and MDS-UPDRS-III $3.13\left(\mathrm{r}_{\mathrm{s}}=0.312, p=0.009\right)$ respectively. One explanation is that F7 may have covered the content reflected by F6 and F8 in a sense.

The present study has some limitations. Firstly, the number of participants were unevenly distributed among groups of different MDS-UPDRS-III 3.13 score, which may affect the statistical results. Secondly, the posture analysis did not consider the torsion problem, therefore it may underestimate the severity of postural abnormalities in some patients. Thirdly, we only evaluated postural abnormalities of PD patients in the standing state and did not reflect the dynamic changes of abnormal posture while walking. These limitations indicate that 
the automated assessment of postural abnormalities in PD patients by artificial intelligence needs to be further improved. More optimized algorithms of machine learning, larger data sets and standardized recording modes will continue to improve the detection and accurate assessment of postural abnormalities in patients with complex patterns.

\section{Conclusions}

From our findings, we demonstrate that automated evaluating system, based on the Kinect v2 sensor and machine learning together with the selected extracted features, represents a valid tool to support the assessment of postural abnormalities in PD. F7 was regarded as a key index to evaluate the severity of camptocormia. In addition, the simple and fast feature of the proposed method support and encourage its practicability in a real clinical scenario. Future work will focus on the application of such method on clinical practice, e.g. to evaluate the effect of certain treatments on PD patients and reveal the dynamic changes of abnormal posture.

\section{Acknowledgements}

Not applicable.

\section{Authors' contributions}

ZYZ, QG and LJJ conceived and designed the study with the help of TYZ. XYS, YJ, YCG, YDL and HPZ developed the algorithm. Data acquisition and analysis was done by $Z Y Z, A L, R H H$, and KWP. RHH was a major contributor in writing the manuscript. All authors read and approved the final manuscript.

\section{Funding}

This study was supported by the National Key Research and Development Program (2018YFC1314700), Sub-project of the Yangtze River Delta Regional Innovation Community Project of Shanghai Municipal Science and Technology Commission (20642430100), Science and Technology Innovation Action Plan of Shanghai Municipal Science and Technology Commission (19441908000), Clinical Technology Innovation Project of Shanghai Shenkang Hospital Development Center (SHDC12020119, SHDC12018X08) and Clinical Technology Innovation Project of Allergan Company (HX2005).

\section{Availability of data and materials}

The datasets used and/or analyzed during the current study are available from the corresponding author on reasonable request.

\section{Declarations}

Ethics approval and consent to participate

The Ethics Committee of Shanghai Tongji Hospital approved all the procedures. Written informed consent was obtained from all the participants.

\section{Consent for publication}

Not applicable.

\section{Competing interests}

The authors declare that they have no competing interests.

\section{Author details}

${ }^{1}$ Neurological Department of Tongji Hospital, Tongji University School of Medicine, Shanghai, China. ${ }^{2}$ F LYTEKK Suzhou Research Institute, E4, Artificial Intelligence Industrial Park, Suzhou Industrial Park, Suzhou, China. ${ }^{3}$ Department of Neurorehabilitation, Yangzhi Rehabilitation Hospital (Shanghai Sunshine Rehabilitation Center), Tongji University School of Medicine, Shanghai, China.
Received: 11 June 2021 Accepted: 11 November 2021

Published online: 04 December 2021

\section{References}

1. Nussbaum RL, Ellis CE. Alzheimer's disease and Parkinson's disease. N Engl J Med. 2003;348(14):1356-64.

2. Tinazzi M, Gandolfi M, Ceravolo R, Capecci M, Andrenelli E, Ceravolo MG, Bonanni L, Onofrj M, Vitale M, Catalan M, et al. Postural Abnormalities in Parkinson's Disease: An Epidemiological and Clinical Multicenter Study. Mov Disord Clin Pract. 2019;6(7):576-85.

3. Pandey S, Kumar H. Assessment of striatal \& postural deformities in patients with Parkinson's disease. Indian J Med Res. 2016;144(5):682-8.

4. Doherty KM, van de Warrenburg BP, Peralta MC, Silveira-Moriyama L, Azulay J-P, Gershanik OS, Bloem BR. Postural deformities in Parkinson's disease. The Lancet Neurology. 2011;10(6):538-49.

5. McFarland C, Wang-Price S, Richard S. Clinical measurements of cervical lordosis using flexirule and inclinometer methods in individuals with and without cervical spine dysfunction: A reliability and validity study. J Back Musculoskelet Rehabil. 2015;28(2):295-302.

6. Tinazzi M, Geroin C, Gandolfi M, Smania N, Tamburin S, Morgante F, Fasano A. Pisa syndrome in Parkinson's disease: An integrated approach from pathophysiology to management. Movement Disorders. 2016;31(12):1785-95.

7. Margraf NG, Wolke R, Granert O, Berardelli A, Bloem BR, Djaldetti R, Espay AJ, Fasano A, Furusawa Y, Giladi N, Hallett M. Consensus for the measurement of the camptocormia angle in the standing patient. Parkinsonism Related Disorders. 2018;52:1-5.

8. Tinazzi M, Gandolfi M, Artusi CA, Lanzafame R, Zanolin E, Ceravolo R, Capecci M, Andrenelli E, Ceravolo MG, Bonanni L, et al. Validity of the wall goniometer as a screening tool to detect postural abnormalities in Parkinson's disease. Parkinsonism Relat Disord. 2019:69:159-65.

9. Goetz CG, Tilley BC, Shaftman SR, Stebbins GT, Fahn S, Martinez-Martin P, Poewe W, Sampaio C, Stern MB, Dodel R, et al. Movement Disorder Society-sponsored revision of the Unified Parkinson's Disease Rating Scale (MDS-UPDRS): scale presentation and clinimetric testing results. Movement Disorders. 2008;23(15):2129-70.

10. Orcioli-Silva D, Beretta VS. Applicability of the Wall Goniometer in Parkinson's disease. Parkinsonism Relat Disord. 2019;69:157-8.

11. Palmerini L, Rocchi L, Mellone S, Valzania F, Chiari L. Feature selection for accelerometer-based posture analysis in Parkinson's disease. IEEE Trans Inform Technol Biomed. 2011;15(3):481-90.

12. Caudron S, Guerraz M, Eusebio A, Gros JP, Azulay JP, Vaugoyeau M. Evaluation of a visual biofeedback on the postural control in Parkinson's disease. Neurophysiologie Clinique. 2014;44(1):77-86.

13. Cancela J, Pastorino M, Tzallas AT, Tsipouras MG, Rigas G, Arredondo MT, Fotiadis DI. Wearability assessment of a wearable system for Parkinson's disease remote monitoring based on a body area network of sensors. Sensors (Basel). 2014;14(9):17235-55.

14. Asakawa T, Sugiyama K, Nozaki T, Sameshima T, Kobayashi S, Wang L, Hong Z, Chen S, Li C, Namba H. Can the latest computerized technologies revolutionize conventional assessment tools and therapies for a neurological disease? The example of Parkinson's disease. Neurol Med Chir (Tokyo). 2019;59(3):69-78.

15. Ledger D, Mccaffrey D: Inside wearables: How the science of human behavior change offers the secret to long-Term engagement. 2014.

16. Espay AJ, Bonato P, Nahab FB, Maetzler W, Dean JM, Klucken J, Eskofier BM, Merola A, Horak F, Lang AE, et al. Technology in Parkinson's disease: challenges and opportunities. Mov Disord. 2016;31(9):1272-82.

17. Han J, Shao L, Xu D, Shotton J. Enhanced computer vision with Microsoft Kinect sensor: a review. IEEE Trans Cybern. 2013;43(5):1318-34.

18. Okada Y, Shibata T, Tamei T, Orito Y, Funaya H. In-home posture evaluation and visual feedback training to improve posture with a kinect-based system in Parkinson's disease. J Novel Physiother. 2014;4(5):232.

19. Giger ML. Machine learning in medical imaging. J Am Coll Radiol. 2018;15(3 Pt B):512-20.

20. Handelman GS, Kok HK, Chandra RV, Razavi AH, Lee MJ, Asadi H. eDoctor: machine learning and the future of medicine. J Intern Med. 2018;284(6):603-19. 
21. Lee S, Mohr NM, Street WN, Nadkarni P. Machine learning in relation to emergency medicine clinical and operational scenarios: an overview. West J Emerg Med. 2019;20(2):219-27.

22. Saber H, Somai M, Rajah GB, Scalzo F, Liebeskind DS. Predictive analytics and machine learning in stroke and neurovascular medicine. Neurol Res. 2019;41(8):681-90.

23. Badillo S, Banfai B, Birzele F, Davydov II, Hutchinson L, Kam-Thong T, Siebourg-Polster J, Steiert B, Zhang JD. An Introduction to Machine Learning. Clin Pharmacol Ther. 2020;107(4):871-85.

24. Dranca L. Using Kinect to classify Parkinson's disease stages related to severity of gait impairment. BMC Bioinform. 2018;19(1):471.

25. Ferraris C, Nerino R, Chimienti A, Pettiti G, Cau N, Cimolin V, Azzaro C, Albani G, Priano L, Mauro A. A Self-Managed System for Automated Assessment of UPDRS Upper Limb Tasks in Parkinson's Disease. Sensors (Basel). 2018;18:10.

26. Arippa F, Pau M, Cimolin V, Stocchi F, Goffredo M, Franceschini M, Condoluci C, De Pandis MF, Galli M. A novel summary kinematic index for postural characterization in subjects with Parkinson's disease. Eur J Phys Rehab Med. 2020;56:2

27. Ferraris C, Nerino R, Chimienti A, Pettiti G, Cau N, Cimolin V, Azzaro C, Priano L, Mauro A. Feasibility of home-based automated assessment of postural instability and lower limb impairments in Parkinson's disease. Sensors (Basel). 2019;19(5):1129.

28. Buongiorno D, Bortone I, Cascarano GD, Trotta GF, Brunetti A, Bevilacqua V. A low-cost vision system based on the analysis of motor features for recognition and severity rating of Parkinson's Disease. BMC Med Inform Decis Mak. 2019;19(Suppl 9):243.

29. Tan D, Pua YH, Balakrishnan S, Scully A, Bower KJ, Prakash KM, Tan EK, Chew JS, Poh E, Tan SB, et al. Automated analysis of gait and modified timed up and go using the Microsoft Kinect in people with Parkinson's disease: associations with physical outcome measures. Med Biol Eng Comput. 2019;57(2):369-77.

30. Bonanni L, Thomas A, Varanese S, Scorrano V, Onofrj M. Botulinum toxin treatment of lateral axial dystonia in Parkinsonism. Mov Disord. 2007;22(14):2097-103.

31. Barone P, Santangelo G, Amboni M, Pellecchia MT, Vitale C. Pisa syndrome in Parkinson's disease and parkinsonism: clinical features, pathophysiology, and treatment. Lancet Neurol. 2016;15(10):1063-74.

32. Koo TK, Li MY. A guideline of selecting and reporting intraclass correlation coefficients for reliability research. J Chiropr Med. 2016;15(2):155-63.

33. Siderowf A, McDermott M, Kieburtz K, Blindauer K, Plumb S, Shoulson I, Parkinson Study G. Test-retest reliability of the unified Parkinson's disease rating scale in patients with early Parkinson's disease: results from a multicenter clinical trial. Movement Disorders. 2002;17(4):758-63.

34. Mao Y, He Y, Liu L, Chen X. Disease classification based on eye movement features with decision tree and random forest. Front Neurosci. 2020;14:798.

35. Panhalkar AR, Doye DD: A novel approach to build accurate and diverse decision tree forest. Evolutionary intelligence 2021:1-15.

36. Clark RA, Pua YH, Bryant AL, Hunt MA. Validity of the Microsoft Kinect for providing lateral trunk lean feedback during gait retraining. Gait Posture. 2013;38(4):1064-6.

37. Clark RA, Pua YH, Fortin K, Ritchie C, Webster KE, Denehy L, Bryant AL. Validity of the Microsoft Kinect for assessment of postural control. Gait Posture. 2012;36(3):372-7.

38. Clark RA, Pua YH, Oliveira CC, Bower KJ, Thilarajah S, McGaw R, Hasanki K, Mentiplay BF. Reliability and concurrent validity of the Microsoft Xbox One Kinect for assessment of standing balance and postural control. Gait Posture. 2015;42(2):210-3

39. Xu H, Yu Y, Zhou Y, Li Y, Du S. Measuring accurate body parameters of dressed humans with large-scale motion using a Kinect sensor. Sensors (Basel). 2013;13(9):11362-84

40. Guerrero C, Uribe-Quevedo A. Kinect-based posture tracking for correcting positions during exercise. Stud Health Technol Inform. 2013;184:158-60.

41. Romano G, Viggiano D. Interception of moving objects in karate: an experimental, marker-free benchmark. Muscles Ligaments Tendons J. 2014;4(2):101-5.

42. Asakawa T, Fang H, Sugiyama K, Nozaki T, Kobayashi S, Hong Z, Suzuki K, Mori N, Yang Y, Hua F, et al. Human behavioral assessments in current research of Parkinson's disease. Neurosci Biobehav Rev. 2016;68:741-72.
43. Asakawa T, Fang H, Sugiyama K, Nozaki T, Hong Z, Yang Y, Hua F, Ding G, Chao D, Fenoy AJ, et al. Animal behavioral assessments in current research of Parkinson's disease. Neurosci Biobehav Rev. 2016;65:63-94.

44. Greene PE, Bressman S. Exteroceptive and interoceptive stimuli in dystonia. Mov Disord. 1998;13(3):549-51.

\section{Publisher's Note}

Springer Nature remains neutral with regard to jurisdictional claims in published maps and institutional affiliations.
Ready to submit your research? Choose BMC and benefit from:

- fast, convenient online submission

- thorough peer review by experienced researchers in your field

- rapid publication on acceptance

- support for research data, including large and complex data types

- gold Open Access which fosters wider collaboration and increased citations

- maximum visibility for your research: over $100 \mathrm{M}$ website views per year

At BMC, research is always in progress.

Learn more biomedcentral.com/submissions 